Onkologie 1994;17(suppl 1):3

\title{
Inhalt, Vol. 17, Supplement 1, 1994
}

\section{Einführung}

Possinger, $\mathrm{K} 5$

Droloxifen erzeugt im Gegensatz zu Tamoxifen keine Lebertumoren an der Ratte

Dahme, E., Rattel, B 6

Droloxifen hemmt das Wachstum und die Proteinsynthese von Brustkrebszellen effektiver als Tamoxifen und Toremifen

Biedermann, E., Loser, R., Hasmann, M 17

Wachstumshemmung von menschlichen Tumorzellen durch intermittierende Behandlung mit Droloxifen

Hasmann, M., Loser, R., Kohr, A., Seibel, K 22

Hemmung der wachstumsfaktorinduzierten Proliferation von MCF-7-Mammakarzinomzellen durch Antiöstrogene und Effekte auf Protoonkogen-Aktivierungen

Küng, W., Wosikowski, K., Hasmann, M., Loser, R., Eppenberger, U 27

Droloxifen bei postmenopausalen Patientinnen mit metastasiertem Mammakarzinom:

Doppelblind randomisierte Phase-П-Studie

Marschner, N., Kreienberg, R., Balas, R., Brandtner, M.,

Schlingensiepen, R., Schumann, S., Büttner, S., Staab, H.J.,

Rauschning, W. $\quad 32$

Droloxifen in der Therapie des metastasierten Mamma-karzinoms: Ergebnisse der frühen

Dosisfindungsstudie

Kreienberg, R 40

Antiöstrogene als primäre Therapie bei älteren Mammakarzinompatientinnen

Serin, D., Krams, M 45

Pharmakokinetik von Droloxifen im Vergleich zu Tamoxifen

Breitbach, G.P., Reister, C, Droege, H, Bastert, G 49

Zusammenfassung

Bastert, G 54 1994;017:3 